\title{
Bimatrix variate beta type IV distribution: relation to Wilks's statistic and bimatrix variate Kummer-beta type IV distribution
}

\author{
BEKKER, A. ${ }^{1}$, ROUX, J.J.J., EHLERS, R. and ARASHI, M.* \\ Department of Statistics, \\ Faculty of Natural and Agricultural Sciences, \\ University of Pretoria, Pretoria, 0002, South Africa \\ *Faculty of Mathematics, \\ Shahrood University of Technology, \\ P. O. Box 316-3619995161, \\ Shahrood, Iran
}

\begin{abstract}
In this paper the bimatrix variate beta type IV distribution is derived from independent Wishart distributed matrix variables. We explore specific properties of this distribution which is then used to derive the exact expressions of the densities of the product and ratio of two dependent Wilks's statistics and to define the bimatrix Kummer-beta type IV distribution.
\end{abstract}

Keywords and phrases-bimatrix variate beta type IV distribution; bimatrix variate Kummer-beta type IV distribution; hypergeometric function of matrix argument; invariant polynomials; moment generating function; Meijer's G-function; Wilks's statistic; Wishart distribution.

2000 Mathematics Subject Classification: primary 62H10; secondary 62E15.

\section{Introduction}

Consider three independent Wishart matrix variables $\boldsymbol{S}_{i} \sim W_{p}\left(n_{i}, \boldsymbol{\Sigma}\right), i=1,2$ and $\boldsymbol{B} \sim W_{p}(m, \boldsymbol{\Sigma})$ and define the transformation

$$
\boldsymbol{X}_{i}=\left(\boldsymbol{I}_{p}+\boldsymbol{B}^{-\frac{1}{2}} \boldsymbol{S}_{i} \boldsymbol{B}^{-\frac{1}{2}}\right)^{-\frac{1}{2}} \boldsymbol{B}^{-\frac{1}{2}} \boldsymbol{S}_{i} \boldsymbol{B}^{-\frac{1}{2}}\left(\boldsymbol{I}_{p}+\boldsymbol{B}^{-\frac{1}{2}} \boldsymbol{S}_{i} \boldsymbol{B}^{-\frac{1}{2}}\right)^{-\frac{1}{2}}
$$

where $\boldsymbol{B}^{\frac{1}{2}} \boldsymbol{B}^{\frac{1}{2}}=\boldsymbol{B}$ and $\mathbf{0}<\boldsymbol{X}_{i}<\boldsymbol{I}_{p}, \quad i=1,2$. In this paper we derive the distribution of $\left(\boldsymbol{X}_{1}, \boldsymbol{X}_{2}\right)$ and refer to it as the bimatrix variate beta type $I V$ distribution. Specific properties are explored and then used as the basis to derive two further important results.

Firstly, it follows from (1) that $\Lambda_{1}=\left|\frac{\boldsymbol{S}_{1}}{\boldsymbol{S}_{1}+\boldsymbol{B}}\right|=\left|\boldsymbol{X}_{1}\right|$ and $\Lambda_{2}=\left|\frac{\boldsymbol{S}_{2}}{\boldsymbol{S}_{2}+\boldsymbol{B}}\right|=\left|\boldsymbol{X}_{2}\right|$, thus $Z_{1}=\left|\boldsymbol{X}_{1} \boldsymbol{X}_{2}\right|=\Lambda_{1} \Lambda_{2}$ and $Z_{2}=\left|\frac{\boldsymbol{X}_{1}}{\boldsymbol{X}_{2}}\right|=\frac{\Lambda_{1}}{\Lambda_{2}}$ are the product and ratio of two dependent Wilks's statistics. We derive $E\left(\left|\boldsymbol{X}_{1}\right|^{h_{1}}\left|\boldsymbol{X}_{2}\right|^{h_{2}}\right)$ for the bimatrix variate beta type IV distribution and use it to obtain exact expressions for the densities of $Z_{1}$ and $Z_{2}$ in terms of Meijer's G-function. The Wilks's statistic is widely used for various statistical tests in multivariate analysis. Exact expressions have been derived for the distribution of the Wilks's statistic (Mathai and Rathie, 1969) and also for the product and ratio of two independent Wilks's statistics (Kshirsagar, 1972; Pham-Gia, 2008).

Secondly, the moment generating function of the bimatrix variate beta type IV distribution is derived and then applied to define the bimatrix variate Kummer-beta type $I V$ distribution. This is an extension of the bimatrix variate beta type $I V$ distribution. In the bivariate case it is an extension of the distribution proposed independently by Libby and Novick (1982), Jones (2001) and Olkin and Liu (2003). Gupta et al. (2001) defined the matrix variate Kummer-Dirichlet type I and type II distributions which reduce

\footnotetext{
${ }^{1}$ Corresponding author:

E-mail:andriette.bekker@up.ac.za Fax:+27 124203440
} 
under certain conditions to the matrix variate Kummer-beta distribution (Nagar and Gupta, 2002) and the matrix variate Kummer-gamma distribution (Nagar and Cardeño, 2001).

The distribution of $\left(\boldsymbol{X}_{1}, \boldsymbol{X}_{2}\right)$ is derived in Section 2 by using the transformation in (1). In Section 3 the product moment of this distribution is applied to obtain the exact expressions for the densities of $Z_{1}=\left|\boldsymbol{X}_{1} \boldsymbol{X}_{2}\right|$ and $Z_{2}=\left|\frac{\boldsymbol{X}_{1}}{\boldsymbol{X}_{2}}\right|$. The moment generating function of the bimatrix beta type IV distribution is derived in Section 4. This result is used to define the bimatrix variate Kummer-beta type $I V$ distribution. In Section 5 the form of the densities of $Z_{1}$ and $Z_{2}$ is illustrated, as well as the effect of the parameters on the conditional moments for the bivariate beta type IV distribution. The form of the density of the bivariate Kummer-beta type $I V$ distribution also receives attention.

\section{Bimatrix variate beta type IV distribution}

In this section the bimatrix variate beta type IV distribution is derived from a transformation of independent Wishart distributed random matrices.

\section{Theorem 1}

Let $\boldsymbol{S}_{1} \sim W_{p}\left(n_{1}, \boldsymbol{\Sigma}\right), \boldsymbol{S}_{2} \sim W_{p}\left(n_{2}, \boldsymbol{\Sigma}\right)$ and $\boldsymbol{B} \sim W_{p}(m, \boldsymbol{\Sigma})$ be independently distributed. Define

$$
\boldsymbol{X}_{i}=\left(\boldsymbol{I}_{p}+\boldsymbol{B}^{-\frac{1}{2}} \boldsymbol{S}_{i} \boldsymbol{B}^{-\frac{1}{2}}\right)^{-\frac{1}{2}} \boldsymbol{B}^{-\frac{1}{2}} \boldsymbol{S}_{i} \boldsymbol{B}^{-\frac{1}{2}}\left(\boldsymbol{I}_{p}+\boldsymbol{B}^{-\frac{1}{2}} \boldsymbol{S}_{i} \boldsymbol{B}^{-\frac{1}{2}}\right)^{-\frac{1}{2}}, i=1,2,
$$

where $\boldsymbol{B}^{\frac{1}{2}} \boldsymbol{B}^{\frac{1}{2}}=\boldsymbol{B}$ and $\mathbf{0}<\boldsymbol{X}_{i}<\boldsymbol{I}_{p}, \quad i=1,2$.

Furthermore, $n_{i}>(p-1), i=1,2$ and $m>(p-1)$.

The joint density of $\left(\boldsymbol{X}_{1}, \boldsymbol{X}_{2}\right)$ is

$$
\begin{aligned}
& \left\{\beta_{p}\left(\frac{n_{1}}{2}, \frac{n_{2}}{2} ; \frac{m}{2}\right)\right\}^{-1}\left\{\prod_{i=1}^{2}\left|\boldsymbol{X}_{i}\right|^{\frac{1}{2} n_{i}-\frac{1}{2}(p+1)}\right\} \\
& \cdot\left|\boldsymbol{I}_{p}-\boldsymbol{X}_{1}\right|^{\frac{1}{2}\left(n_{2}+m\right)-\frac{1}{2}(p+1)}\left|\boldsymbol{I}_{p}-\boldsymbol{X}_{2}\right|^{\frac{1}{2}\left(n_{1}+m\right)-\frac{1}{2}(p+1)}\left|\boldsymbol{I}_{p}-\boldsymbol{X}_{1} \boldsymbol{X}_{2}\right|^{-\frac{1}{2}\left(n_{1}+n_{2}+m\right)}
\end{aligned}
$$

where $\beta_{p}\left(\frac{n_{1}}{2}, \frac{n_{2}}{2} ; \frac{m}{2}\right)=\frac{\Gamma_{p}\left(\frac{n_{1}}{2}\right) \Gamma_{p}\left(\frac{n_{2}}{2}\right) \Gamma_{p}\left(\frac{m}{2}\right)}{\Gamma_{p}\left(\frac{n_{1}+n_{2}+m}{2}\right)}$, and $\Gamma_{p}(\cdot)$ is the multivariate gamma function.

The density in (3) is that of the bimatrix variate beta type $I V$ distribution and is denoted as $\left(\boldsymbol{X}_{1}, \boldsymbol{X}_{2}\right) \sim$ $B B_{p}^{I V}\left(\frac{n_{1}}{2}, \frac{n_{2}}{2} ; \frac{m}{2}\right)$.

\section{Proof:}

The joint density of $\left(\boldsymbol{S}_{1}, \boldsymbol{S}_{2}, \boldsymbol{B}\right)$ is given by

$$
K\left\{\prod_{i=1}^{2}\left[\operatorname{etr}\left(-\frac{1}{2} \boldsymbol{\Sigma}^{-1} \boldsymbol{S}_{i}\right)\left|\boldsymbol{S}_{i}\right|^{\frac{1}{2}\left(n_{i}-p-1\right)}\right]\right\}\left[\operatorname{etr}\left(-\frac{1}{2} \boldsymbol{\Sigma}^{-1} \boldsymbol{B}\right)|\boldsymbol{B}|^{\frac{1}{2}(m-p-1)}\right]
$$

where $K^{-1}=2^{\frac{1}{2}\left(n_{1}+n_{2}+m\right) p} \Gamma_{p}\left(\frac{n_{1}}{2}\right) \Gamma_{p}\left(\frac{n_{2}}{2}\right) \Gamma_{p}\left(\frac{m}{2}\right)|\boldsymbol{\Sigma}|^{\frac{1}{2}\left(n_{1}+n_{2}+m\right)}$.

Making the transformations

$$
\boldsymbol{X}_{i}=\left(\boldsymbol{I}_{p}+\boldsymbol{B}^{-\frac{1}{2}} \boldsymbol{S}_{i} \boldsymbol{B}^{-\frac{1}{2}}\right)^{-\frac{1}{2}} \boldsymbol{B}^{-\frac{1}{2}} \boldsymbol{S}_{i} \boldsymbol{B}^{-\frac{1}{2}}\left(\boldsymbol{I}_{p}+\boldsymbol{B}^{-\frac{1}{2}} \boldsymbol{S}_{i} \boldsymbol{B}^{-\frac{1}{2}}\right)^{-\frac{1}{2}}, i=1,2,
$$

and

$$
\boldsymbol{Z}_{i}=\boldsymbol{B}^{-\frac{1}{2}} \boldsymbol{S}_{i} \boldsymbol{B}^{-\frac{1}{2}}, i=1,2
$$


gives $\boldsymbol{X}_{i}=\left(\boldsymbol{I}_{p}+\boldsymbol{Z}_{i}\right)^{-1} \boldsymbol{Z}_{i}$ since $\boldsymbol{Z}_{i}$ commutes with any rational function of $\boldsymbol{Z}_{i}$. The Jacobian of the transformation (see Gupta and Nagar, 2000) is

$$
\begin{aligned}
J\left(\boldsymbol{S}_{1}, \boldsymbol{S}_{2} \rightarrow \boldsymbol{X}_{1}, \boldsymbol{X}_{2}\right) & =\left\{\prod_{i=1}^{2} J\left(\boldsymbol{S}_{i} \rightarrow \boldsymbol{Z}_{i}\right) J\left(\boldsymbol{Z}_{i} \rightarrow \boldsymbol{X}_{i}\right)\right\} \\
& =|\boldsymbol{B}|^{(p+1)} \prod_{i=1}^{2}\left|\boldsymbol{I}_{p}-\boldsymbol{X}_{i}\right|^{-(p+1)} .
\end{aligned}
$$

Substituting this in (4) gives the joint density of $\left(\boldsymbol{X}_{1}, \boldsymbol{X}_{2}, \boldsymbol{B}\right)$ as

$$
\begin{aligned}
f\left(\boldsymbol{X}_{1}, \boldsymbol{X}_{2}, \boldsymbol{B}\right)= & K\left\{\prod_{i=1}^{2}\left|\boldsymbol{X}_{i}\right|^{\frac{1}{2} n_{i}-\frac{1}{2}(p+1)}\right\}\left\{\prod_{i=1}^{2}\left|\boldsymbol{I}_{p}-\boldsymbol{X}_{i}\right|^{-\frac{1}{2} n_{i}-\frac{1}{2}(p+1)}\right\} \\
& \cdot|\boldsymbol{B}|^{\frac{1}{2}\left(n_{1}+n_{2}+m\right)-\frac{1}{2}(p+1)} \operatorname{etr}\left\{-\frac{1}{2} \boldsymbol{B}^{\frac{1}{2}} \boldsymbol{\Sigma}^{-1} \boldsymbol{B}^{\frac{1}{2}}\left[\boldsymbol{I}_{p}+\sum_{i=1}^{2} \boldsymbol{X}_{i}\left(\boldsymbol{I}_{p}-\boldsymbol{X}_{i}\right)^{-1}\right]\right\} .
\end{aligned}
$$

Making the transformation $\boldsymbol{X}_{i} \rightarrow \boldsymbol{H} \boldsymbol{X}_{i} \boldsymbol{H}^{\prime}, i=1,2$ and $\boldsymbol{B} \rightarrow \boldsymbol{H} \boldsymbol{B} \boldsymbol{H}^{\prime}$ where $\boldsymbol{H}(p \times p)$ is orthogonal (Díaz-García and Gutiérrez-Jáimez, 2006) and substituting in (5) gives

\section{$f\left(\boldsymbol{H} \boldsymbol{X}_{1} \boldsymbol{H}^{\prime}, \boldsymbol{H} \boldsymbol{X}_{2} \boldsymbol{H}^{\prime}, \boldsymbol{H} \boldsymbol{B} \boldsymbol{H}^{\prime}\right)$}

$$
\begin{aligned}
= & K\left\{\prod_{i=1}^{2}\left|\boldsymbol{X}_{i}\right|^{\frac{1}{2} n_{i}-\frac{1}{2}(p+1)}\right\}\left\{\prod_{i=1}^{2}\left|\boldsymbol{I}_{p}-\boldsymbol{X}_{i}\right|^{-\frac{1}{2} n_{i}-\frac{1}{2}(p+1)}\right\} \\
& \cdot|\boldsymbol{B}|^{\frac{1}{2}\left(n_{1}+n_{2}+m\right)-\frac{1}{2}(p+1)} \operatorname{etr}\left\{-\frac{1}{2}\left(\boldsymbol{H} \boldsymbol{B}^{\frac{1}{2}} \boldsymbol{H}^{\prime}\right) \boldsymbol{\Sigma}^{-1}\left(\boldsymbol{H} \boldsymbol{B}^{\frac{1}{2}} \boldsymbol{H}^{\prime}\right)\left[\boldsymbol{I}_{p}+\sum_{i=1}^{2} \boldsymbol{H} \boldsymbol{X}_{i}\left(\boldsymbol{I}_{p}-\boldsymbol{X}_{i}\right)^{-1} \boldsymbol{H}^{\prime}\right]\right\}
\end{aligned}
$$

We consider the symmetrized density function of $\left(\boldsymbol{X}_{1}, \boldsymbol{X}_{2}\right)$ defined by Greenacre (1973), that is $f_{S}\left(\boldsymbol{X}_{1}, \boldsymbol{X}_{2}\right)=\int_{\boldsymbol{B}>\mathbf{0}} \int_{O(p)} f\left(\boldsymbol{H} \boldsymbol{X}_{1} \boldsymbol{H}^{\prime}, \boldsymbol{H} \boldsymbol{X}_{2} \boldsymbol{H}^{\prime}, \boldsymbol{H} \boldsymbol{B} \boldsymbol{H}^{\prime}\right) \mathrm{d} \boldsymbol{H} \mathrm{d} \boldsymbol{B}$ where $\boldsymbol{H}(p \times p)$ is orthogonal and $\mathrm{d} \boldsymbol{H}$ is the normalized invariant measure on $O(p)$. Note that $\mathrm{d} \boldsymbol{B}=\mathrm{d} \boldsymbol{H} \boldsymbol{B} \boldsymbol{H}^{\prime} \quad$ (Díaz-García and GutiérrezJáimez, 2006).

Then

$$
\begin{aligned}
& f_{S}\left(\boldsymbol{X}_{1}, \boldsymbol{X}_{2}\right) \\
& =\quad K\left\{\prod_{i=1}^{2}\left|\boldsymbol{X}_{i}\right|^{\frac{1}{2} n_{i}-\frac{1}{2}(p+1)}\right\}\left\{\prod_{i=1}^{2}\left|\boldsymbol{I}_{p}-\boldsymbol{X}_{i}\right|^{-\frac{1}{2} n_{i}-\frac{1}{2}(p+1)}\right\} \\
& \quad \cdot \int_{\boldsymbol{B}>\mathbf{0}}|\boldsymbol{B}|^{\frac{1}{2}\left(n_{1}+n_{2}+m\right)-\frac{1}{2}(p+1)} \int_{O(p)} \operatorname{etr}\left\{-\frac{1}{2} \boldsymbol{H}^{\prime} \boldsymbol{\Sigma}^{-1} \boldsymbol{H} \boldsymbol{B}^{\frac{1}{2}}\left[\boldsymbol{I}_{p}+\sum_{i=1}^{2} \boldsymbol{X}_{i}\left(\boldsymbol{I}_{p}-\boldsymbol{X}_{i}\right)^{-1}\right] \boldsymbol{B}^{\frac{1}{2}}\right\} \mathrm{d} \boldsymbol{H} \mathrm{d} \boldsymbol{B} .
\end{aligned}
$$

It follows from Ehlers, Bekker and Roux (2009, Lemma 5, page 114) that

$$
\begin{aligned}
& \int_{O(p)} \operatorname{etr}\left\{-\frac{1}{2} \boldsymbol{H}^{\prime} \boldsymbol{\Sigma}^{-1} \boldsymbol{H} \boldsymbol{B}^{\frac{1}{2}}\left[\boldsymbol{I}_{p}+\sum_{i=1}^{2} \boldsymbol{X}_{i}\left(\boldsymbol{I}_{p}-\boldsymbol{X}_{i}\right)^{-1}\right] \boldsymbol{B}^{\frac{1}{2}}\right\} \mathrm{d} \boldsymbol{H} \\
& =\int_{O(p)} \operatorname{etr}\left\{-\frac{1}{2}\left[\boldsymbol{I}_{p}+\sum_{i=1}^{2} \boldsymbol{X}_{i}\left(\boldsymbol{I}_{p}-\boldsymbol{X}_{i}\right)^{-1}\right]^{\frac{1}{2}} \boldsymbol{H}^{\prime} \boldsymbol{\Sigma}^{-1} \boldsymbol{H}\left[\boldsymbol{I}_{p}+\sum_{i=1}^{2} \boldsymbol{X}_{i}\left(\boldsymbol{I}_{p}-\boldsymbol{X}_{i}\right)^{-1}\right]^{\frac{1}{2}} \boldsymbol{B}\right\} \mathrm{d} \boldsymbol{H} .
\end{aligned}
$$

Form the above, changing the order of integration in (6) and integrating with respect to $\boldsymbol{B}$ as well as using Gupta and Nagar (2000, Equation 1.4.6, page 19) gives

$$
\begin{aligned}
f_{S}\left(\boldsymbol{X}_{1}, \boldsymbol{X}_{2}\right)= & \left\{\beta_{p}\left(\frac{n_{1}}{2}, \frac{n_{2}}{2} ; \frac{m}{2}\right)\right\}^{-1}\left\{\prod_{i=1}^{2}\left|\boldsymbol{X}_{i}\right|^{\frac{1}{2} n_{i}-\frac{1}{2}(p+1)}\right\}\left\{\prod_{i=1}^{2}\left|\boldsymbol{I}_{p}-\boldsymbol{X}_{i}\right|^{-\frac{1}{2} n_{i}-\frac{1}{2}(p+1)}\right\} \\
& \cdot \int_{O(p)}\left|\boldsymbol{I}_{p}+\boldsymbol{H} \sum_{i=1}^{2} \boldsymbol{X}_{i}\left(\boldsymbol{I}_{p}-\boldsymbol{X}_{i}\right)^{-1} \boldsymbol{H}^{\prime}\right|^{-\frac{1}{2}\left(n_{1}+n_{2}+m\right)} \mathrm{d} \boldsymbol{H} .
\end{aligned}
$$


Since $f_{S}\left(\boldsymbol{X}_{1}, \boldsymbol{X}_{2}\right)=\int_{O(p)} f\left(\boldsymbol{H} \boldsymbol{X}_{1} \boldsymbol{H}^{\prime}, \boldsymbol{H} \boldsymbol{X}_{2} \boldsymbol{H}^{\prime}\right) \mathrm{d} \boldsymbol{H}$, it follows from (7) that

$$
f\left(\boldsymbol{H} \boldsymbol{X}_{1} \boldsymbol{H}^{\prime}, \boldsymbol{H} \boldsymbol{X}_{2} \boldsymbol{H}^{\prime}\right)
$$

$$
\begin{aligned}
= & \left\{\beta_{p}\left(\frac{n_{1}}{2}, \frac{n_{2}}{2} ; \frac{m}{2}\right)\right\}^{-1}\left\{\prod_{i=1}^{2}\left|\boldsymbol{X}_{i}\right|^{\frac{1}{2} n_{i}-\frac{1}{2}(p+1)}\right\}\left\{\prod_{i=1}^{2}\left|\boldsymbol{I}_{p}-\boldsymbol{X}_{i}\right|^{-\frac{1}{2} n_{i}-\frac{1}{2}(p+1)}\right\} \\
& \cdot\left|\boldsymbol{I}_{p}+\boldsymbol{H} \sum_{i=1}^{2} \boldsymbol{X}_{i}\left(\boldsymbol{I}_{p}-\boldsymbol{X}_{i}\right)^{-1} \boldsymbol{H}\right|^{-\frac{1}{2}\left(n_{1}+n_{2}+m\right)} .
\end{aligned}
$$

Making the transformation $\boldsymbol{H} \boldsymbol{X}_{i} \boldsymbol{H}^{\prime} \rightarrow \boldsymbol{X}_{i}, \quad i=1,2$ and rewriting $\left|\boldsymbol{I}_{p}+\sum_{i=1}^{2} \boldsymbol{X}_{i}\left(\boldsymbol{I}_{p}-\boldsymbol{X}_{i}\right)^{-1}\right|$ as

$$
\begin{aligned}
& \left|\boldsymbol{I}_{p}+\boldsymbol{X}_{2}\left(\boldsymbol{I}_{p}-\boldsymbol{X}_{2}\right)^{-1}-\boldsymbol{X}_{2}\left(\boldsymbol{I}_{p}-\boldsymbol{X}_{2}\right)^{-1} \boldsymbol{X}_{1}\right|\left|\boldsymbol{I}_{p}-\boldsymbol{X}_{1}\right|^{-1} \\
& =\left|\boldsymbol{I}_{p}-\boldsymbol{X}_{2}\left(\boldsymbol{I}_{p}-\boldsymbol{X}_{2}\right)^{-1} \boldsymbol{X}_{1}+\boldsymbol{X}_{2}\left(\boldsymbol{I}_{p}-\boldsymbol{X}_{2}\right)^{-1} \boldsymbol{X}_{1} \boldsymbol{X}_{2}\right|\left|\boldsymbol{I}_{p}-\boldsymbol{X}_{1}\right|^{-1}\left|\boldsymbol{I}_{p}-\boldsymbol{X}_{2}\right|^{-1} \\
& =\left|\boldsymbol{I}_{p}-\boldsymbol{X}_{1} \boldsymbol{X}_{2}\right|\left|\boldsymbol{I}_{p}-\boldsymbol{X}_{1}\right|^{-1}\left|\boldsymbol{I}_{p}-\boldsymbol{X}_{2}\right|^{-1}
\end{aligned}
$$

gives the result.

\section{Remarks}

1. If $\left(\boldsymbol{X}_{1}, \boldsymbol{X}_{2}\right) \sim B B_{p}^{I V}\left(\frac{n_{1}}{2}, \frac{n_{2}}{2} ; \frac{m}{2}\right)$ with density function given in (3), then

(a) the marginal density functions are matrix variate beta type I, $\boldsymbol{X}_{i} \sim B_{p}^{I}\left(\frac{n_{i}}{2} ; \frac{m}{2}\right), \quad i=1,2$;

(b) the conditional density of $\boldsymbol{X}_{2} \mid \boldsymbol{X}_{1}$ is given by

$$
\begin{array}{r}
\left\{\beta_{p}\left(\frac{n_{2}}{2} ; \frac{n_{1}+m}{2}\right)\right\}^{-1}\left|\boldsymbol{I}_{p}-\boldsymbol{X}_{1}\right|^{\frac{1}{2} n_{2}}\left|\boldsymbol{X}_{2}\right|^{\frac{1}{2} n_{2}-\frac{1}{2}(p+1)}\left|\boldsymbol{I}_{p}-\boldsymbol{X}_{2}\right|^{\frac{1}{2}\left(n_{1}+m\right)-\frac{1}{2}(p+1)}\left|\boldsymbol{I}_{p}-\boldsymbol{X}_{1} \boldsymbol{X}_{2}\right|^{-\frac{1}{2}\left(n_{1}+n_{2}+m\right),}, \\
\mathbf{0}<\boldsymbol{X}_{2}<\boldsymbol{I}_{p} .
\end{array}
$$

2. The matrix variate Dirichlet type IV, denoted as $\left(\boldsymbol{X}_{1}, \ldots, \boldsymbol{X}_{r}\right) \sim D_{p}^{I V}\left(\frac{n_{1}}{2}, \ldots, \frac{n_{r}}{2} ; \frac{m}{2}\right)$, results by extending (2) to $r$ independent Wishart matrix variables, $\boldsymbol{S}_{i} \sim W_{p}\left(n_{i}, \boldsymbol{\Sigma}\right), i=1, \ldots, r$, all independent of $\boldsymbol{B} \sim W_{p}(m, \boldsymbol{\Sigma})$. The joint density of $\left(\boldsymbol{X}_{1}, \ldots, \boldsymbol{X}_{r}\right)$ is given by

$$
\begin{aligned}
f\left(\boldsymbol{X}_{1}, \ldots, \boldsymbol{X}_{r}\right) & \\
= & \left\{\beta_{p}\left(\frac{n_{1}}{2}, \ldots, \frac{n_{r}}{2} ; \frac{m}{2}\right)\right\}^{-1}\left\{\prod_{i=1}^{r}\left|\boldsymbol{X}_{i}\right|^{\frac{1}{2} n_{i}-\frac{1}{2}(p+1)}\right\} \\
& \cdot\left\{\prod_{i=1}^{r}\left|\boldsymbol{I}_{p}-\boldsymbol{X}_{i}\right|^{-\frac{1}{2} n_{i}-\frac{1}{2}(p+1)}\right\}\left|\boldsymbol{I}_{p}+\sum_{i=1}^{r} \boldsymbol{X}_{i}\left(\boldsymbol{I}_{p}-\boldsymbol{X}_{i}\right)^{-1}\right|^{-\frac{1}{2}\left(n_{1}+\cdots+n_{r}+m\right)}
\end{aligned}
$$

where $\mathbf{0}<\boldsymbol{X}_{i}<\mathbf{I}_{p}, i=1, \ldots, r$.

\section{Distribution of product and ratio of dependent Wilks's sta- tistics}

In this section we derive the exact expressions for the density functions of $Z_{1}=\left|\boldsymbol{X}_{1} \boldsymbol{X}_{2}\right|=\Lambda_{1} \Lambda_{2}$ and $Z_{2}=\left|\frac{\boldsymbol{X}_{1}}{\boldsymbol{X}_{2}}\right|=\frac{\Lambda_{1}}{\Lambda_{2}}$, the product and ratio of two dependent Wilks's statistics, in terms of Meijer's G-function. The proofs are based on the Mellin transforms of the density functions of $Z_{1}$ and $Z_{2}$, and their inverse Mellin transforms. 


\section{$\underline{\text { Lemma } 1}$}

If $\left(\boldsymbol{X}_{1}, \boldsymbol{X}_{2}\right) \sim B B_{p}^{I V}\left(\frac{n_{1}}{2}, \frac{n_{2}}{2} ; \frac{m}{2}\right)$ with density function given in (3), then

$$
\begin{aligned}
E\left[\left|\boldsymbol{X}_{1}\right|^{h_{1}}\left|\boldsymbol{X}_{2}\right|^{h_{2}}\right]= & \frac{\beta_{p}\left(\frac{n_{1}}{2}+h_{1} ; \frac{n_{2}+m}{2}\right) \beta_{p}\left(\frac{n_{2}}{2}+h_{2} ; \frac{n_{1}+m}{2}\right)}{\beta_{p}\left(\frac{n_{1}}{2}, \frac{n_{2}}{2} ; \frac{m}{2}\right)} \\
& \cdot{ }_{3} F_{2}\left(\frac{n_{1}}{2}+h_{1}, \frac{n_{2}}{2}+h_{2}, \frac{n_{1}+n_{2}+m}{2} ; \frac{n_{1}+n_{2}+m}{2}+h_{1}, \frac{n_{1}+n_{2}+m}{2}+h_{2} ; \boldsymbol{I}_{p}\right)
\end{aligned}
$$

where ${ }_{3} F_{2}(\cdot)$ is a hypergeometric function of matrix argument, (see Muirhead, 1982, Definition 7.3.1, page 258).

\section{Proof:}

Using Gupta and Nagar (2000, Equations 1.6.6 and 1.6.8, page 36) the result in (9) follows.

\section{Theorem 2}

Let $\left(\boldsymbol{X}_{1}, \boldsymbol{X}_{2}\right) \sim B B_{p}^{I V}\left(\frac{n_{1}}{2}, \frac{n_{2}}{2} ; \frac{m}{2}\right)$ and $Z_{1}=\left|\boldsymbol{X}_{1} \boldsymbol{X}_{2}\right|=\Lambda_{1} \Lambda_{2}$.

The density function of $Z_{1}$, the product of two dependent Wilks statistics, is given by

$$
\frac{\Gamma_{p}\left(\frac{n_{1}+m}{2}\right) \Gamma_{p}\left(\frac{n_{2}+m}{2}\right)}{\Gamma_{p}\left(\frac{n_{1}}{2}\right) \Gamma_{p}\left(\frac{n_{2}}{2}\right) \Gamma_{p}\left(\frac{m}{2}\right)} \sum_{k=0}^{\infty} \sum_{\kappa} \frac{C_{\kappa}\left(\boldsymbol{I}_{p}\right)}{k !} \Gamma_{p}\left(\frac{n_{1}+n_{2}+m}{2}, \kappa\right) G_{2 p, 2 p}^{2 p, 0}\left(\left.z_{1}\right|_{b_{1}, \ldots, b_{2 p}} ^{a_{1}, \ldots, a_{2 p}}\right), \quad 0<z_{1}<1
$$

where $a_{j}= \begin{cases}\frac{n_{1}+n_{2}+m}{2}-1+k_{(j+1) / 2}-\frac{1}{4}(j-1) & \text { for } j=1,3,5, \ldots, 2 p-1 \\ \frac{n_{1}+n_{2}+m}{2}-1+k_{j / 2}-\frac{1}{4}(j-2) & \text { for } j=2,4,6, \ldots, 2 p,\end{cases}$

and $b_{j}= \begin{cases}\frac{n_{1}}{2}-1+k_{(j+1) / 2}-\frac{1}{4}(j-1) & \text { for } j=1,3,5, \ldots, 2 p-1 \\ \frac{n_{2}}{2}-1+k_{j / 2}-\frac{1}{4}(j-2) & \text { for } j=2,4,6, \ldots, 2 p .\end{cases}$

Furthermore ${ }^{C_{\kappa}}\left(\boldsymbol{I}_{p}\right)$ is the zonal polynomial of $\boldsymbol{I}_{p}$ corresponding to $\kappa$ and $\Gamma_{p}(a, \kappa)=\pi^{\frac{1}{4} p(1-p)} \prod_{j=1}^{p} \Gamma\left[a+k_{j}-\frac{1}{2}(j-1)\right]$ (see Gupta and Nagar, 2000).

\section{Proof:}

It follows from (9) that

$$
E\left(Z_{1}^{h-1}\right)=\frac{\Gamma_{p}\left(\frac{n_{1}+m}{2}\right) \Gamma_{p}\left(\frac{n_{2}+m}{2}\right)}{\Gamma_{p}\left(\frac{n_{1}}{2}\right) \Gamma_{p}\left(\frac{n_{2}}{2}\right) \Gamma_{p}\left(\frac{m}{2}\right)} \sum_{k=0}^{\infty} \sum_{\kappa} \frac{C_{\kappa}\left(\boldsymbol{I}_{p}\right)}{k !} \Gamma_{p}\left(\frac{n_{1}+n_{2}+m}{2}, \kappa\right) \frac{\prod_{j=1}^{2 p} \Gamma\left(b_{j}+h\right)}{\prod_{j=1}^{2 p} \Gamma\left(a_{j}+h\right)},
$$

where $a_{j}$ and $b_{j}$ are as defined above.

Using Meijer's G-function (Mathai, 1993, Definition 2.1, page 60) and the inverse Mellin transform (Mathai, 1993, Definition 1.8, page 23), the desired result (10) follows.

\section{Theorem 3}

Let $\left(\boldsymbol{X}_{1}, \boldsymbol{X}_{2}\right) \sim B B_{p}^{I V}\left(\frac{n_{1}}{2}, \frac{n_{2}}{2} ; \frac{m}{2}\right)$ and $Z_{2}=\left|\frac{\boldsymbol{X}_{1}}{\boldsymbol{X}_{2}}\right|=\frac{\Lambda_{1}}{\Lambda_{2}}$.

The density function of $Z_{2}$, the ratio of two dependent Wilks's statistics, is given by

$$
\frac{\Gamma_{p}\left(\frac{n_{1}+m}{2}\right) \Gamma_{p}\left(\frac{n_{2}+m}{2}\right)}{\Gamma_{p}\left(\frac{n_{1}}{2}\right) \Gamma_{p}\left(\frac{n_{2}}{2}\right) \Gamma_{p}\left(\frac{m}{2}\right)} \sum_{k=0}^{\infty} \sum_{\kappa} \frac{C_{\kappa}\left(\boldsymbol{I}_{p}\right)}{k !} \Gamma_{p}\left(\frac{n_{1}+n_{2}+m}{2}, \kappa\right) G_{2 p, 2 p}^{p, p}\left(\left.z_{2}\right|_{b_{1}, \ldots, b_{2 p}} ^{a_{1}, \ldots, a_{2 p}}\right) \quad \text { for } z_{2}>0
$$

where $a_{j}= \begin{cases}-\frac{n_{2}}{2}-k_{j}+\frac{1}{2}(j-1) & \text { for } j=1,2, \ldots, p \\ \frac{n_{1}+n_{2}+m}{2}-1+k_{j-p}-\frac{1}{2}(j-p-1) & \text { for } j=p+1, p+2, \ldots, 2 p,\end{cases}$

and $\quad b_{j}= \begin{cases}\frac{n_{1}}{2}-1+k_{j}-\frac{1}{2}(j-1) & \text { for } j=1,2, \ldots, p \\ -\frac{n_{1}+n_{2}+m}{2}-k_{j-p}+\frac{1}{2}(j-p-1) & \text { for } j=p+1, p+2, \ldots, 2 p .\end{cases}$ 
Furthermore $C_{\kappa}\left(\boldsymbol{I}_{p}\right)$ is the zonal polynomial of $\boldsymbol{I}_{p}$ corresponding to $\kappa$ and $\Gamma_{p}(a, \kappa)=\pi^{\frac{1}{4} p(1-p)} \prod_{j=1}^{p} \Gamma\left[a+k_{j}-\frac{1}{2}(j-1)\right] \quad$ (see Gupta and Nagar, 2000).

\section{Proof:}

It follows from (9) that

$E\left(Z_{2}^{h-1}\right)=\frac{\Gamma_{p}\left(\frac{n_{1}+m}{2}\right) \Gamma_{p}\left(\frac{n_{2}+m}{2}\right)}{\Gamma_{p}\left(\frac{n_{1}}{2}\right) \Gamma_{p}\left(\frac{n_{2}}{2}\right) \Gamma_{p}\left(\frac{m}{2}\right)} \cdot \sum_{k=0}^{\infty} \sum_{\kappa} \frac{C_{\kappa}\left(\boldsymbol{I}_{p}\right)}{k !} \frac{\Gamma_{p}\left(\frac{n_{1}+n_{2}+m}{2}, \kappa\right) \Gamma_{p}\left(\frac{n_{1}}{2}+h-1, \kappa\right) \Gamma_{p}\left(\frac{n_{2}}{2}-h+1, \kappa\right)}{\Gamma_{p}\left(\frac{n_{1}+n_{2}+m}{2}+h-1, \kappa\right) \Gamma_{p}\left(\frac{n_{1}+n_{2}+m}{2}-h+1, \kappa\right)}$.

where $a_{j}$ and $b_{j}$ are defined above.

Using Meijer's G-function, the inverse Mellin transform and Mathai (1993, Equation 2.2.4, page 72), the required result (11) follows.

\section{Remark}

In the case when $p=1$, the results in (10) and (11) simplifies to the densities of the product and ratio of correlated beta type I random variables obtained by Nagar et al. (2009).

\section{Bimatrix variate Kummer-beta type IV distribution}

In this section we obtain the moment generating function of the bimatrix variate beta type IV distribution and define the bimatrix variate Kummer-beta type IV distribution.

\section{Lemma 2}

Suppose that $\boldsymbol{X}=\left[\boldsymbol{X}_{1}: \boldsymbol{X}_{2}\right]$ and $\boldsymbol{T}=\left[\boldsymbol{T}_{1}: \boldsymbol{T}_{2}\right]$. Then the moment generating function of the bimatrix variate beta type IV distribution given in (3) is given by

$$
\begin{aligned}
\mathcal{M}(\boldsymbol{T})= & \left\{\beta_{p}\left(\frac{n_{1}}{2}, \frac{n_{2}}{2} ; \frac{m}{2}\right)\right\}^{-1} \sum_{k=0}^{\infty} \sum_{t=0}^{\infty} \sum_{z=0}^{\infty} \sum_{\kappa} \sum_{\tau} \sum_{\zeta} \sum_{\delta \in \kappa \cdot \tau} \sum_{\gamma \in \kappa \cdot \zeta} \frac{\left(\frac{n_{1}+n_{2}+m}{2}\right)_{\kappa}}{k ! t ! z !} \\
& \cdot \frac{g_{\kappa, \tau}^{\delta}\left(\frac{n_{2}}{2}\right)_{\delta} \beta_{p}\left(\frac{n_{2}}{2}, \frac{n_{1}+m}{2}\right)}{\left(\frac{n_{1}+n_{2}+m}{2}\right)_{\delta}} \frac{f_{\kappa, \zeta}^{\gamma}\left(\frac{n_{1}}{2}\right)_{\gamma} \beta_{p}\left(\frac{n_{1}}{2}, \frac{n_{2}+m}{2}\right)}{\left(\frac{n_{1}+n_{2}+m}{2}\right)_{\gamma}} \\
& \cdot \frac{C_{\zeta}\left(\boldsymbol{T}_{1}\right) C_{\tau}\left(\boldsymbol{T}_{2}\right) C_{\delta}\left(\boldsymbol{I}_{p}\right) C_{\gamma}\left(\boldsymbol{I}_{p}\right)}{C_{\kappa}\left(\boldsymbol{I}_{p}\right) C_{\tau}\left(\boldsymbol{I}_{p}\right) C_{\zeta}\left(\boldsymbol{I}_{p}\right)} .
\end{aligned}
$$

Proof:

By definition, the moment generating function of $\boldsymbol{X}$ is given by

$$
\begin{aligned}
& \mathcal{M}(\boldsymbol{T}) \\
& =\quad E[\operatorname{etr}(\boldsymbol{T X})] \\
& =\left\{\beta_{p}\left(\frac{n_{1}}{2}, \frac{n_{2}}{2} ; \frac{m}{2}\right)\right\}^{-1} \int_{\mathbf{0}<\boldsymbol{X}_{1}<\boldsymbol{I}_{p}} \int_{\mathbf{0}<\boldsymbol{X}_{2}<\boldsymbol{I}_{p}}\left|\boldsymbol{X}_{1}\right|^{\frac{1}{2} n_{1}-\frac{1}{2}(p+1)}\left|\boldsymbol{X}_{2}\right|^{\frac{1}{2} n_{2}-\frac{1}{2}(p+1)}\left|\boldsymbol{I}_{p}-\boldsymbol{X}_{1}\right|^{\frac{1}{2}\left(n_{2}+m\right)-\frac{1}{2}(p+1)} \\
& \cdot\left|\boldsymbol{I}_{p}-\boldsymbol{X}_{2}\right|^{\frac{1}{2}\left(n_{1}+m\right)-\frac{1}{2}(p+1)}\left|\boldsymbol{I}_{p}-\boldsymbol{X}_{1} \boldsymbol{X}_{2}\right|^{-\frac{1}{2}\left(n_{1}+n_{2}+m\right)} \operatorname{etr}\left(\boldsymbol{T}_{1} \boldsymbol{X}_{1}\right) \operatorname{etr}\left(\boldsymbol{T}_{2} \boldsymbol{X}_{2}\right) d \boldsymbol{X}_{2} d \boldsymbol{X}_{1} \\
& =\left\{\beta_{p}\left(\frac{n_{1}}{2}, \frac{n_{2}}{2} ; \frac{m}{2}\right)\right\}^{-1} \int_{\boldsymbol{X}_{1}}\left|\boldsymbol{X}_{1}\right|^{\frac{1}{2} n_{1}-\frac{1}{2}(p+1)}\left|\boldsymbol{I}_{p}-\boldsymbol{X}_{1}\right|^{\frac{1}{2}\left(n_{2}+m\right)-\frac{1}{2}(p+1)} \operatorname{etr}\left(\boldsymbol{T}_{1} \boldsymbol{X}_{1}\right) A\left(\boldsymbol{X}_{1}\right) d \boldsymbol{X}_{1} \text {, }
\end{aligned}
$$




$$
\begin{aligned}
A\left(\boldsymbol{X}_{1}\right) & \int_{\boldsymbol{X}_{2}}\left|\boldsymbol{X}_{2}\right|^{\frac{1}{2} n_{2}-\frac{1}{2}(p+1)}\left|\boldsymbol{I}_{p}-\boldsymbol{X}_{2}\right|^{\frac{1}{2}\left(n_{1}+m\right)-\frac{1}{2}(p+1)} \operatorname{etr}\left(\boldsymbol{T}_{2} \boldsymbol{X}_{2}\right)\left|\boldsymbol{I}_{p}-\boldsymbol{X}_{1} \boldsymbol{X}_{2}\right|^{-\frac{1}{2}\left(n_{1}+n_{2}+m\right)} \mathrm{d} \boldsymbol{X}_{2} \\
= & \sum_{k=0}^{\infty} \sum_{t=0}^{\infty} \sum_{\kappa} \sum_{\tau} \frac{\left(\frac{n_{1}+n_{2}+m}{2}\right)_{\kappa}}{k ! t !} \int_{\boldsymbol{X}_{2}}\left|\boldsymbol{X}_{2}\right|^{\frac{1}{2} n_{2}-\frac{1}{2}(p+1)}\left|\boldsymbol{I}_{p}-\boldsymbol{X}_{2}\right|^{\frac{1}{2}\left(n_{1}+m\right)-\frac{1}{2}(p+1)} C_{\kappa}\left(\boldsymbol{X}_{1} \boldsymbol{X}_{2}\right) C_{\tau}\left(\boldsymbol{T}_{2} \boldsymbol{X}_{2}\right) \mathrm{d} \boldsymbol{X}_{2} \\
= & \sum_{k=0}^{\infty} \sum_{t=0}^{\infty} \sum_{\kappa} \sum_{\tau} \frac{\left(\frac{n_{1}+n_{2}+m}{2}\right)_{\kappa}}{k ! t !} \\
& \cdot \int_{\boldsymbol{X}_{2}}\left|\boldsymbol{X}_{2}\right|^{\frac{1}{2} n_{2}-\frac{1}{2}(p+1)}\left|\boldsymbol{I}_{p}-\boldsymbol{X}_{2}\right|^{\frac{1}{2}\left(n_{1}+m\right)-\frac{1}{2}(p+1)} C_{\tau}\left(\boldsymbol{T}_{2} \boldsymbol{X}_{2}\right) \int_{O(p)} C_{\kappa}\left(\boldsymbol{H}^{\prime} \boldsymbol{X}_{1} \boldsymbol{H} \boldsymbol{X}_{2}\right) \mathrm{d} \boldsymbol{H} \mathrm{d} \boldsymbol{X}_{2} .
\end{aligned}
$$

It follows from Muirhead (1982, Theorem 7.2.5, page 243 and Theorem 7.2.10, page 254) and Chikuse (1981, Equation 2.7) that

$$
\begin{aligned}
A\left(\boldsymbol{X}_{1}\right) & =\sum_{k=0}^{\infty} \sum_{t=0}^{\infty} \sum_{\kappa} \sum_{\tau} \frac{\left(\frac{n_{1}+n_{2}+m}{2}\right)_{\kappa}}{k ! t !} \frac{C_{\kappa}\left(\boldsymbol{X}_{1}\right)}{C_{\kappa}\left(\boldsymbol{I}_{p}\right)} \int_{\boldsymbol{X}_{2}}\left|\boldsymbol{X}_{2}\right|^{\frac{1}{2} n_{2}-\frac{1}{2}(p+1)}\left|\boldsymbol{I}_{p}-\boldsymbol{X}_{2}\right|^{\frac{1}{2}\left(n_{1}+m\right)-\frac{1}{2}(p+1)} C_{\kappa}\left(\boldsymbol{X}_{2}\right) C_{\tau}\left(\boldsymbol{T}_{2} \boldsymbol{X}_{2}\right) \mathrm{d} \boldsymbol{X}_{2} \\
= & \sum_{k=0}^{\infty} \sum_{t=0}^{\infty} \sum_{\kappa} \sum_{\tau} \frac{\left(\frac{n_{1}+n_{2}+m}{2}\right)_{\kappa}}{k ! t !} \frac{C_{\kappa}\left(\boldsymbol{X}_{1}\right) C_{\tau}\left(\boldsymbol{T}_{2}\right)}{C_{\kappa}\left(\boldsymbol{I}_{p}\right) C_{\tau}\left(\boldsymbol{I}_{p}\right)} \int_{\boldsymbol{X}_{2}}\left|\boldsymbol{X}_{2}\right|^{\frac{1}{2} n_{2}-\frac{1}{2}(p+1)}\left|\boldsymbol{I}_{p}-\boldsymbol{X}_{2}\right|^{\frac{1}{2}\left(n_{1}+m\right)-\frac{1}{2}(p+1)} C_{\kappa}\left(\boldsymbol{X}_{2}\right) C_{\tau}\left(\boldsymbol{X}_{2}\right) \mathrm{d} \boldsymbol{X}_{2} \\
= & \sum_{k=0}^{\infty} \sum_{t=0}^{\infty} \sum_{\kappa} \sum_{\tau} \frac{\left(\frac{n_{1}+n_{2}+m}{2}\right)_{\kappa}}{k ! t !} \frac{C_{\kappa}\left(\boldsymbol{X}_{1}\right) C_{\tau}\left(\boldsymbol{T}_{2}\right)}{C_{\kappa}\left(\boldsymbol{I}_{p}\right) C_{\tau}\left(\boldsymbol{I}_{p}\right)} \\
& \cdot \sum_{\delta \in \kappa \cdot \tau} g_{\kappa, \tau}^{\delta} \int_{\boldsymbol{X}_{2}}\left|\boldsymbol{X}_{2}\right|^{\frac{1}{2} n_{2}-\frac{1}{2}(p+1)}\left|\boldsymbol{I}_{p}-\boldsymbol{X}_{2}\right|^{\frac{1}{2}\left(n_{1}+m\right)-\frac{1}{2}(p+1)} C_{\delta}\left(\boldsymbol{X}_{2}\right) \mathrm{d} \boldsymbol{X}_{2} \\
= & \sum_{k=0}^{\infty} \sum_{t=0}^{\infty} \sum_{\kappa} \sum_{\tau} \sum_{\delta \in \kappa \cdot \tau} \frac{\left(\frac{n_{1}+n_{2}+m}{2}\right)_{\kappa}}{k ! t !} \frac{g_{\kappa, \tau}^{\delta}\left(\frac{n_{2}}{2}\right)_{\delta} \beta_{p}\left(\frac{n_{2}}{2}, \frac{n_{1}+m}{2}\right)}{\left(\frac{n_{1}+n_{2}+m}{2}\right)_{\delta}} \frac{C_{\kappa}\left(\boldsymbol{X}_{1}\right) C_{\tau}\left(\boldsymbol{T}_{2}\right) C_{\delta}\left(\boldsymbol{I}_{p}\right)}{C_{\kappa}\left(\boldsymbol{I}_{p}\right) C_{\tau}\left(\boldsymbol{I}_{p}\right)}
\end{aligned}
$$

Substituting (14) in (13) and continuing in the same way, the result follows.

\section{Definition}

The $p \times p$ symmetric positive definite random matrices $\boldsymbol{X}_{1}$ and $\boldsymbol{X}_{2}$ on the unit $p$-sphere are said to have the bimatrix variate Kummer-beta type $I V$ distribution with parameters $n_{1}, n_{2}, m$ and $\mathbf{\Psi}$, denoted by $\left(\boldsymbol{X}_{1}, \boldsymbol{X}_{2}\right) \sim B K B_{p}^{I V}\left(\frac{n_{1}}{2}, \frac{n_{2}}{2} ; \frac{m}{2} ; \boldsymbol{\Psi}\right)$ if their joint density is given by

$$
\begin{aligned}
& K\left|\boldsymbol{X}_{1}\right|^{\frac{1}{2} n_{1}-\frac{1}{2}(p+1)}\left|\boldsymbol{X}_{2}\right|^{\frac{1}{2} n_{2}-\frac{1}{2}(p+1)}\left|\boldsymbol{I}_{p}-\boldsymbol{X}_{1}\right|^{\frac{1}{2}\left(n_{2}+m\right)-\frac{1}{2}(p+1)} \\
& \cdot\left|\boldsymbol{I}_{p}-\boldsymbol{X}_{2}\right|^{\frac{1}{2}\left(n_{1}+m\right)-\frac{1}{2}(p+1)}\left|\boldsymbol{I}_{p}-\boldsymbol{X}_{1} \boldsymbol{X}_{2}\right|^{-\frac{1}{2}\left(n_{1}+n_{2}+m\right)} \operatorname{etr}\left[-\boldsymbol{\Psi}\left(\boldsymbol{X}_{1}+\boldsymbol{X}_{2}\right)\right]
\end{aligned}
$$

where

$$
\begin{aligned}
K^{-1}= & \mathcal{M}(-\boldsymbol{\Psi}:-\boldsymbol{\Psi}) \\
= & \left\{\beta_{p}\left(\frac{n_{1}}{2}, \frac{n_{2}}{2} ; \frac{m}{2}\right)\right\}^{-1} \sum_{k=0}^{\infty} \sum_{t=0}^{\infty} \sum_{z=0}^{\infty} \sum_{\kappa} \sum_{\tau} \sum_{\zeta} \sum_{\delta \in \kappa \cdot \tau} \sum_{\gamma \in \kappa \cdot \zeta} \frac{\left(\frac{n_{1}+n_{2}+m}{2}\right)_{\kappa}}{k ! t ! z !} \\
& \cdot \frac{g_{\kappa, \tau}^{\delta}\left(\frac{n_{2}}{2}\right)_{\delta} \beta_{p}\left(\frac{n_{2}}{2}, \frac{n_{1}+m}{2}\right)}{\left(\frac{n_{1}+n_{2}+m}{2}\right)_{\delta}} \frac{f_{\kappa, \zeta}^{\gamma}\left(\frac{n_{1}}{2}\right)_{\gamma} \beta_{p}\left(\frac{n_{1}}{2}, \frac{n_{2}+m}{2}\right)}{\left(\frac{n_{1}+n_{2}+m}{2}\right)_{\gamma}} \\
& \cdot \frac{C_{\zeta}(-\boldsymbol{\Psi}) C_{\tau}(-\boldsymbol{\Psi}) C_{\delta}\left(\boldsymbol{I}_{p}\right) C_{\gamma}\left(\boldsymbol{I}_{p}\right)}{C_{\kappa}\left(\boldsymbol{I}_{p}\right) C_{\tau}\left(\boldsymbol{I}_{p}\right) C_{\zeta}\left(\boldsymbol{I}_{p}\right)} .
\end{aligned}
$$

The expression for $K$ follows directly from (12). 


\section{Shape analysis}

In Section 5.1 we illustrate the effect of the parameters on the shape of the densities of $Z_{1}=\left|\boldsymbol{X}_{1} \boldsymbol{X}_{2}\right|$ and $Z_{2}=\left|\frac{\boldsymbol{X}_{1}}{\boldsymbol{X}_{2}}\right|$. The effect of the parameters on the conditional moments of the bivariate beta type IV variables as well as on the form of the bivariate Kummer-beta type IV distribution are illustrated in Sections 5.2 and 5.3 respectively. Only the two cases $p=2$ and $p=1$ are studied, for products and ratios of dependent Wilks's statistics, and conditional densities respectively. For the Kummer-beta type IV distribution, again only the unidimensional case is studied. The cases $p>2$ are much more complicated to deal with numerically.

\subsection{Distributions of product and ratio of dependent Wilks's statistics}

We consider the bimatrix case with $p=2$ where the density function of $Z_{1}=\left|\boldsymbol{X}_{1} \boldsymbol{X}_{2}\right|=\Lambda_{1} \Lambda_{2} \quad$ (see (10)) simplifies to

$$
f\left(z_{1}\right)=\frac{\Gamma_{2}\left(\frac{n_{1}+m}{2}\right) \Gamma_{2}\left(\frac{n_{2}+m}{2}\right)}{\Gamma_{2}\left(\frac{n_{1}}{2}\right) \Gamma_{2}\left(\frac{n_{2}}{2}\right) \Gamma_{2}\left(\frac{m}{2}\right)} \sum_{k=0}^{\infty} \sum_{\kappa} \frac{C_{\kappa}\left(\boldsymbol{I}_{2}\right)}{k !} \Gamma_{2}\left(\frac{n_{1}+n_{2}+m}{2}, \kappa\right) G_{4,4}^{4,0}\left(\left.z_{1}\right|_{b_{1}, b_{2}, b_{3}, b_{4}} ^{a_{1}, a_{2}, a_{3}, a_{4}}\right), \quad 0<z_{1}<1
$$

where

$a_{1}=\frac{n_{1}+n_{2}+m}{2}-1+k_{1}, a_{2}=\frac{n_{1}+n_{2}+m}{2}-1+k_{1}, a_{3}=\frac{n_{1}+n_{2}+m}{2}-1+k_{2}-\frac{1}{2}$ and $a_{4}=\frac{n_{1}+n_{2}+m}{2}-1+k_{2}-\frac{1}{2}$;

$b_{1}=\frac{n_{1}}{2}-1+k_{1}, b_{2}=\frac{n_{2}}{2}-1+k_{1}, b_{3}=\frac{n_{1}}{2}-1+k_{2}-\frac{1}{2}$ and $b_{4}=\frac{n_{2}}{2}-1+k_{2}-\frac{1}{2}$.

Figure 1 illustrates the shape of $f\left(z_{1}\right)$ for increasing values of $n_{2}$ and $m$. We note that as $n_{2}$ increases the density shifts towards larger values of $z_{1}$. The opposite happens when $m$ increases.

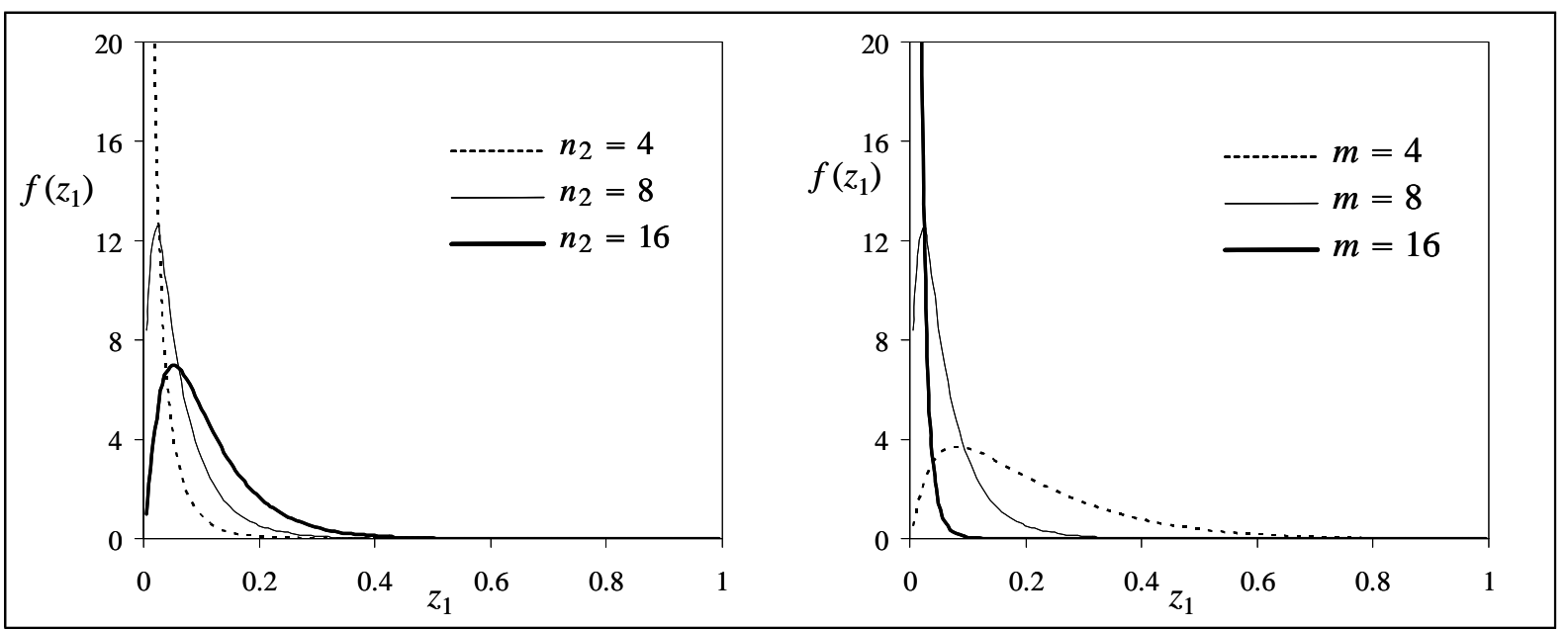

Figure 1: $\quad$ Density function of $Z_{1}=\left|\boldsymbol{X}_{1} \boldsymbol{X}_{2}\right|=\Lambda_{1} \Lambda_{2}$ for

(i) increasing values of $n_{2},\left(\boldsymbol{X}_{1}, \boldsymbol{X}_{2}\right) \sim B B_{2}^{I V}\left(4, \frac{n_{2}}{2} ; 4\right)$ and

(ii) increasing values of $m,\left(\boldsymbol{X}_{1}, \boldsymbol{X}_{2}\right) \sim B B_{2}^{I V}\left(4,4 ; \frac{m}{2}\right)$.

Similarly for $p=2$, the density function of $Z_{2}=\frac{\Lambda_{1}}{\Lambda_{2}}$ given in (11) simplifies to

$$
f\left(z_{2}\right)=\frac{\Gamma_{2}\left(\frac{n_{1}+m}{2}\right) \Gamma_{2}\left(\frac{n_{2}+m}{2}\right)}{\Gamma_{2}\left(\frac{n_{1}}{2}\right) \Gamma_{2}\left(\frac{n_{2}}{2}\right) \Gamma_{2}\left(\frac{m}{2}\right)} \sum_{k=0}^{\infty} \sum_{\kappa} \frac{C_{\kappa}\left(\boldsymbol{I}_{2}\right)}{k !} \Gamma_{2}\left(\frac{n_{1}+n_{2}+m}{2}, \kappa\right) G_{4,4}^{2,2}\left(\left.z_{2}\right|_{b_{1}, b_{2}, b_{3}, b_{4}} ^{a_{1}, a_{2}, a_{3}, a_{4}}\right), \quad z_{2}>0
$$

where

$a_{1}=-\frac{n_{2}}{2}-k_{1}, a_{2}=-\frac{n_{2}}{2}-k_{2}+\frac{1}{2}, a_{3}=\frac{n_{1}+n_{2}+m}{2}-1+k_{1}$ and $a_{4}=\frac{n_{1}+n_{2}+m}{2}-1+k_{2}-\frac{1}{2} ;$

$b_{1}=\frac{n_{1}}{2}-1+k_{1}, b_{2}=\frac{n_{1}}{2}-1+k_{2}-\frac{1}{2}, b_{3}=\frac{2}{\frac{n_{1}+n_{2}+m}{2}}-k_{1}$ and $b_{4}=-\frac{n_{1}^{2}+n_{2}+m}{2}-k_{2}+\frac{1}{2}$.

Figure 2 shows that graphs of $f\left(z_{2}\right)$ for increasing values of $n_{2}$ and $m$. As $n_{2}$ increases the density shifts towards smaller values of $z_{2}$ and the spread of the density decreases. The parameter $n_{1}$ has the opposite effect. As $m$ increases the density shifts towards smaller values of $z_{2}$ and the spread of the density increases. 


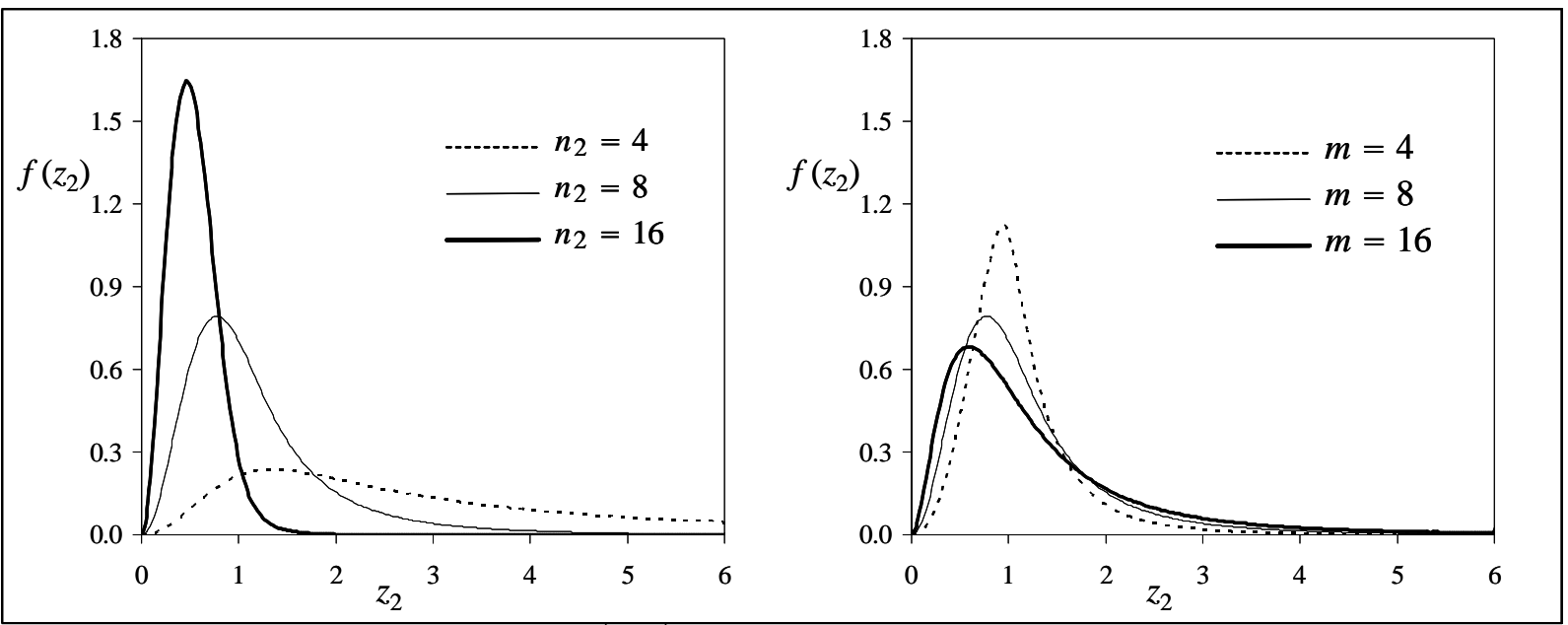

Figure 2: $\quad$ Density function of $Z_{2}=\left|\frac{\boldsymbol{X}_{1}}{\boldsymbol{X}_{2}}\right|=\frac{\Lambda_{1}}{\Lambda_{2}}$ for

(i) increasing values of $n_{2},\left(\boldsymbol{X}_{1}, \boldsymbol{X}_{2}\right) \sim B B_{2}^{I V}\left(4, \frac{n_{2}}{2} ; 4\right)$ and

(ii) increasing values of $m,\left(\boldsymbol{X}_{1}, \boldsymbol{X}_{2}\right) \sim B B_{2}^{I V}\left(4,4 ; \frac{m}{2}\right)$.

Nagar et al. (2009) illustrated the effect of the parameters on the density of the product of correlated beta variables, i.e. $Z_{1}=X_{1} X_{2}$. There are some algorithms available for calculating such functions as (15) and (16) and facilitating the use of these distributions (see Gutierrez et al. (2000) and Koev and Edelman (2006)). There are also mathematical packages, such as Maple and Mathematica for computing and drawing densities in terms of Meijer's G-function. In this paper we used simulations to give graphical representations of the densities of $Z_{1}$ and $Z_{2}$.

\subsection{Effect of parameters on the conditional moments of the bivariate beta type IV distribution}

In the case where $p=1$ the conditional density of $X_{2}$ given $X_{1}$ in (8) is given by

$f\left(x_{2} \mid x_{1}\right)=\left\{\beta\left(\frac{n_{2}}{2} ; \frac{n_{1}+m}{2}\right)\right\}^{-1}\left(1-x_{1}\right)^{\frac{1}{2} n_{2}} x_{2}^{\frac{1}{2} n_{2}-1}\left(1-x_{2}\right)^{\frac{1}{2}\left(n_{1}+m\right)-1}\left(1-x_{1} x_{2}\right)^{-\frac{1}{2}\left(n_{1}+n_{2}+m\right)}, 0<x_{2}<1$.

In Figure 3 the graphs of $E\left(X_{2} \mid X_{1}=x_{1}\right)$ and $\operatorname{var}\left(X_{2} \mid X_{1}=x_{1}\right)$ show the underlying structure of (17) for increasing values of $m$. As $m$ increases whilst all the other parameters are held constant, $E\left(X_{2} \mid X_{1}=x_{1}\right)$ becomes smaller. Similar graphs for increasing values of $n_{2}$ are given in Figure 4 . The conditional expected value, $E\left(X_{2} \mid X_{1}=x_{1}\right)$, increases as $n_{2}$ increases. The parameter $n_{1}$ has the same effect as $m$ on $E\left(X_{2} \mid X_{1}=x_{1}\right)$ and $\operatorname{var}\left(X_{2} \mid X_{1}=x_{1}\right)$ as can be seen from (17). A detailed discussion of the bivariate case is given by Olkin and Liu (2003).

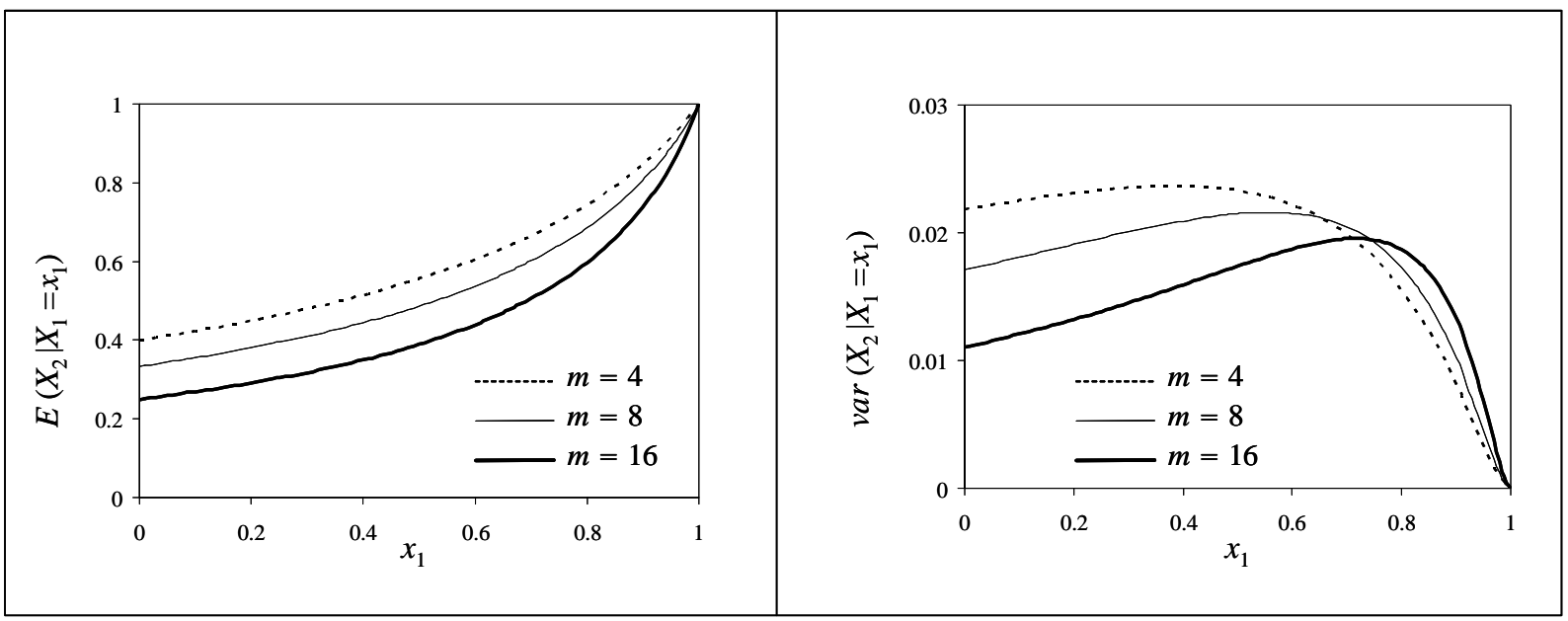

Figure 3: $\quad$ Effect of $m$ on $E\left(X_{2} \mid X_{1}=x_{1}\right)$ and $\operatorname{var}\left(X_{2} \mid X_{1}=x_{1}\right),\left(X_{1}, X_{2}\right) \sim B B^{I V}\left(4,4 ; \frac{m}{2}\right)$ 


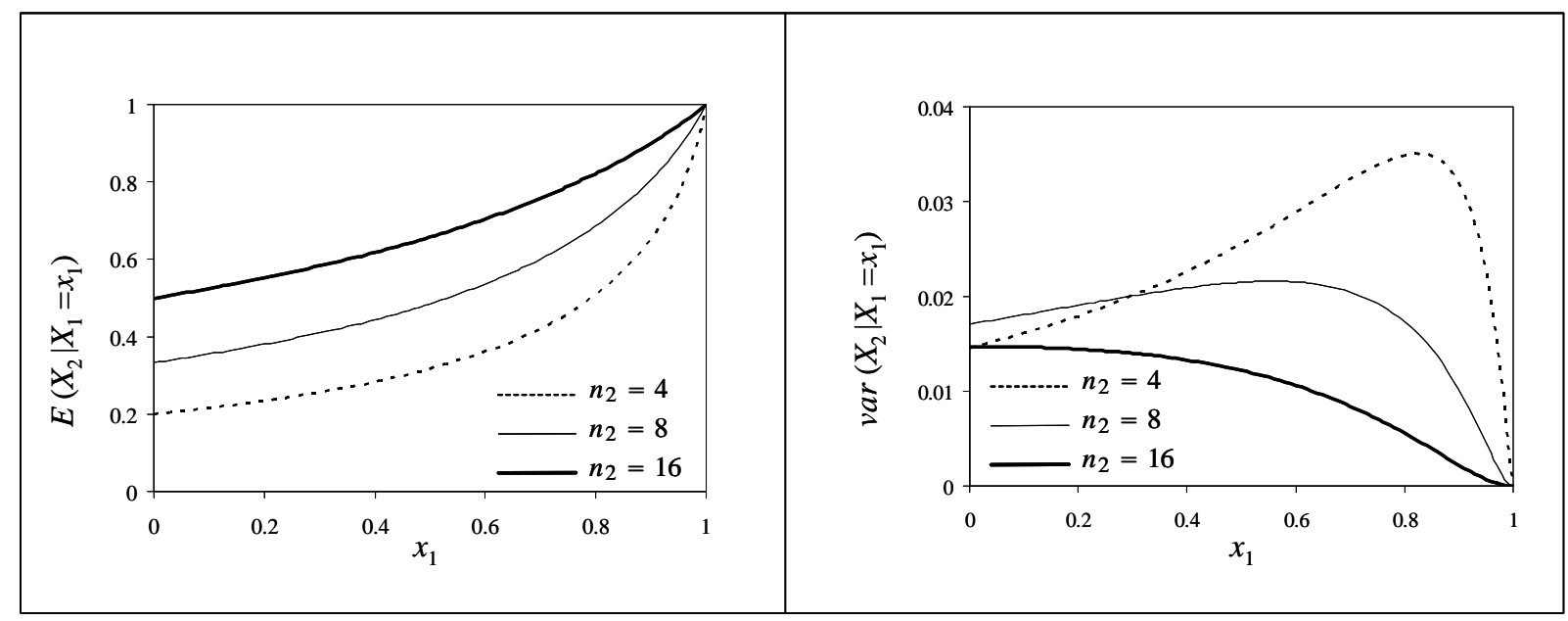

Figure 4: $\quad$ Effect of $n_{2}$ on $E\left(X_{2} \mid X_{1}=x_{1}\right)$ and $\operatorname{var}\left(X_{2} \mid X_{1}=x_{1}\right),\left(X_{1}, X_{2}\right) \sim B B^{I V}\left(4, \frac{n_{2}}{2} ; 4\right)$

\subsection{Bivariate Kummer-beta type IV distribution}

In the case where $p=1$ the density of the bivariate Kummer-beta type $I V$ distribution is given by

$f\left(x_{1}, x_{2}\right)=K x_{1}^{\frac{1}{2} n_{1}-1} x_{2}^{\frac{1}{2} n_{2}-1}\left(1-x_{1}\right)^{\frac{1}{2}\left(n_{2}+m\right)-1}\left(1-x_{2}\right)^{\frac{1}{2}\left(n_{1}+m\right)-1}\left(1-x_{1} x_{2}\right)^{-\frac{1}{2}\left(n_{1}+n_{2}+m\right)} \exp \left[-\psi\left(x_{1}+x_{2}\right)\right]$,

where $0<x_{1}<1$ and $0<x_{2}<1$. The normalizing constant is

$$
\begin{aligned}
K^{-1}= & \frac{\Gamma\left(\frac{n_{1}+m}{2}\right) \Gamma\left(\frac{n_{2}+m}{2}\right)}{\Gamma\left(\frac{n_{1}+n_{2}+m}{2}\right)} \sum_{k=0}^{\infty} \frac{1}{k !} \frac{\Gamma\left(\frac{n_{1}}{2}+k\right) \Gamma\left(\frac{n_{2}}{2}+k\right)}{\Gamma\left(\frac{n_{1}+n_{2}+m}{2}+k\right)} \\
& \cdot{ }_{1} F_{1}\left(\frac{n_{1}}{2}+k ; \frac{n_{1}+n_{2}+m}{2}+k ;-\psi\right){ }_{1} F_{1}\left(\frac{n_{2}}{2}+k ; \frac{n_{1}+n_{2}+m}{2}+k ;-\psi\right)
\end{aligned}
$$

where ${ }_{1} F_{1}(\cdot)$ is the confluent hypergeometric function with scalar argument. The distribution is denoted as $\left(X_{1}, X_{2}\right) \sim B K B^{I V}\left(\frac{n_{1}}{2}, \frac{n_{2}}{2} ; \frac{m}{2} ; \psi\right)$. Figure 5 illustrates the effect of the parameter $\psi$ on this density function. Note that if $\psi=0$ the density simplifies to the bivariate beta type IV (Jones, 2001).
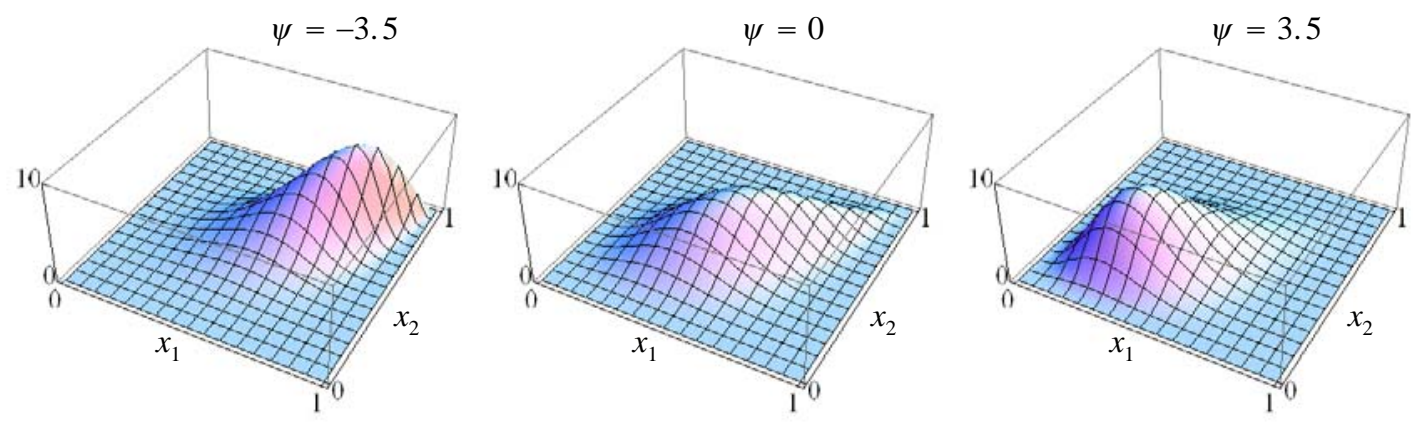

Figure 5: $\quad$ Effect of $\psi$ on $f\left(x_{1}, x_{2}\right),\left(X_{1}, X_{2}\right) \sim B K B^{I V}(4,4 ; 4 ; \psi)$

\section{Conclusion}

In this paper we introduced the bimatrix variate beta type IV distribution and used it to derive the exact expressions of the densities of the product and ratio of two dependent Wilks's statistics. We also defined the bimatrix variate Kummer-beta type IV distribution which followed from the moment 
generating function of the bimatrix variate beta type IV distribution. The effect of the parameters on the shape of the densities were also illustrated. The availability of closed form expressions for the densities of the product and ratio of two dependent Wilks's statistics and the newly proposed bimatrix variate Kummer-beta type IV distribution should stimulate further research and applications.

\section{Acknowledgement}

The authors thank anonymous referees for several comments that have helped in improving the paper. This work is based upon research supported by the National Research Foundation, South Africa (FA2007043000003) and the Research and Development Programme (University of Pretoria).

\section{References}

1. Chikuse, Y. (1981). Distributions of some matrix variates and latent roots in multivariate BehrensFisher discriminant analysis, The Annals of Statistics, 9 (2), 401-407.

2. Díaz-García, J.A. and Gutiérrez-Jáimez, R. (2006). Doubly noncentral matrix variate beta distribution, Comunicación Técnica, No. I-06-08 (PE/CIMAT), Guanajuato, México.

3. Ehlers, R., Bekker, A. and Roux, J.J.J. (2009).The central and non-central matrix variate Dirichlet type III distribution. South African Statistical Journal, 43 (2), 97-116.

4. Greenacre, M.J. (1973). Symmetrized multivariate distributions, South African Statistical Journal, 7, 95-101.

5. Gupta, A.K., Cardeño, L. and Nagar, D.K. (2001). Matrix variate Kummer-Dirichlet distributions, J. Applied Math., 1:3, 117-139.

6. Gupta, A.K. and Nagar, D.K. (2000). Matrix Variate Distributions, Chapman \& Hall/CRC, Boca Raton.

7. Gutiérrez, R., Rodriguez, J. and Sáez, A.J. (2000). Approximation of hypergeometric functions with matricial argument through their development in series of zonal polynomials, Electron. Trans. Numer. Anal., 11, 121-130.

8. Jones, M.C. (2001). Multivariate $t$ and beta distributions associated with the multivariate $F$ distribution. Metrika, 54, 215-231.

9. Koev, P. and Edelman, A. (2006). The efficient evaluation of the hypergeometric function of matrix argument, Math. Comp., 75, 833-846.

10. Kshirsagar, A.M.(1972). Multivariate Analysis, Marcel Dekker, New York.

11. Libby, D.L. and Novick, M.R. (1982). Multivariate generalized beta distributions with applications to utility assessment, Journal of Educational Statistics, 7(4), 271-294.

12. Mathai, A.M. (1993). A Handbook of Generalized Special Functions for Statistical and Physical Sciences, Clarendon Press, Oxford.

13. Mathai, A.M. and Rathie, P.N. (1969). The exact distribution of Wilks' criterion, The Annals of Mathematical Statistics, 42, 3, 1010-1019.

14. Muirhead, R.J. (1982). Aspects of Multivariate Statistical Theory, John Wiley \& Sons, New York.

15. Nagar, D.K. and Cardeño, L. (2001). Matrix variate Kummer-gamma distribution, Random Oper. and Stoch. Equ., 9(3), 207-218.

16. Nagar, D.K. and Gupta, A.K. (2002). Matrix variate Kummer-beta distribution, J. Australian Math. Soc., 73, 11-25. 
17. Nagar, D.K., Orozco-Castañeda, J.M. and Gupta, A.K., (2009). Product and quotient of correlated beta variables, Applied Mathematics Letters, 22, 105-109.

18. Olkin, I. and Liu, R. (2003). A bivariate beta distribution, Statistics and Probability Letters, 62, 407-412.

19. Pham-Gia, T. (2008). Exact distribution of the generalized Wilks's statistic and applications, Journal of Multivariate Analysis, 99, 1698-1716. 